\section{Different diagnostic strategies for different settings}

\section{Ferdinando Petrazzuoli}

Department of Clinical Sciences in Malmö, Centre for Primary Health Care Research, Lund University, Malmö, Sweden

\begin{abstract}
Diagnostic strategies should always take into consideration the setting in which they are administered. The predictive value, positive or negative of a clinical sign or diagnostic test has a different weight in family medicine than in the hospital setting. Many tests have not been formally evaluated in primary care; unfortunately, very often low prevalence settings are used to conduct screening in these populations, and often result in unrealistically high prevalence estimates for chronic disease and these results are then used to conclude that General Practitioners are not good at detecting diseases. In primary care, the prevalence and incidence of disease differs from what appears in the hospital setting, and severe disease occurs less frequently in general practice than in hospital because there is no preventive selection. This requires a specific probability-based decision-making process, based by the knowledge of patients and the community. In primary care, the diagnostic strategy should begin with complaints and symptoms and address uncertainty and complexity, using step-by-step strategies, including watchful waiting, presumptive symptomatic treatment, and focusing on low-tech strategies.
\end{abstract}

\section{The diagnostic process}

There are 2 fundamental strategies in the diagnostic process in Medicine. (Unpublished data: Terry Shaneyfelt, https: //ebmteacher.com/open-teaching-resources/ accessed 28/11/2020): i) pattern recognition: e.g. shingles, where the presence of specific signs and symptoms is pathognomonic of the disease; ii) probabilistic: e.g. deep vein thrombosis, where the probability of a disease is influenced by the diagnostic test accuracy. ${ }^{1}$

The pre-test probability is the probability that a patient is affected by a disease, prior to any further diagnostic studies. In the probabilistic diagnostic paradigm, we start form the pre-test probability and after the test result we have a revised probability (post-test probability). Table 1 shows how can we estimate the pre-test probability.

\section{When to use a supplementary diagnostic test?}

Figure 1 shows the diagnostic process that should guide us in establishing a diagnosis. If further testing is needed, then the next step is to choose a diagnostic test. Because there are often multiple tests that could be ordered for a given disease we need to pick the right one for the situation.

\section{How to choose a diagnostic test?}

Before ordering a test, doctors need to think carefully of the pre-test probability. What do we want to do with a test? Rule in
Correspondence: Ferdinando Petrazzuoli, Center for Primary Health Care Research, Department of Clinical Sciences, Lund University, Box 50332, 202 13, Malmö, Sweden.

Tel.: +4640391363 - Fax: +4640391370 .

E-mail: ferdinando.petrazzuoli@med.lu.se

Key words: Diagnostic process; sensitivity; specificity; likelihood ratio; primary care.

Conflict of interests: the author declares no potential conflict of interests.

Received for publication: 29 November 2020 Accepted for publication: 17 February 2021.

This work is licensed under a Creative Commons Attribution-NonCommercial 4.0 International License (CC BY-NC 4.0).

${ }^{\circ}$ Copyright: the Author(s), 2021

Licensee PAGEPress, Italy

Geriatric Care 2021; 7:9538

doi:10.4081/gc.2021.9538

a disease or rule out a disease? In order to do this, doctors need to compare the operating characteristics of a test before selecting one, and above all, to think about what they will do with the results of a test.

\section{Comparing the operating characteristics of a test before selecting one}

Table 2 illustrates the role of testing. Do we need a sensitive or a specific test? It depends on the role of testing (rule in or rule out).

When we want to rule in a disease, we need a specific test, while when they want

Table 1. Estimating pre-test probability.

1. From direct studies on disease probability. (e.g. probability of have coronary disease in patient presenting with chest pain in an emergency room)

2. Validated clinical prediction rules (e.g. Wells score for Deep Vein Thrombosis (DVT), CHA DS -VASc Score for Atrial Fibrillation Stroke Risk.

3. Clinical experience and judgment. (Guess estimation)

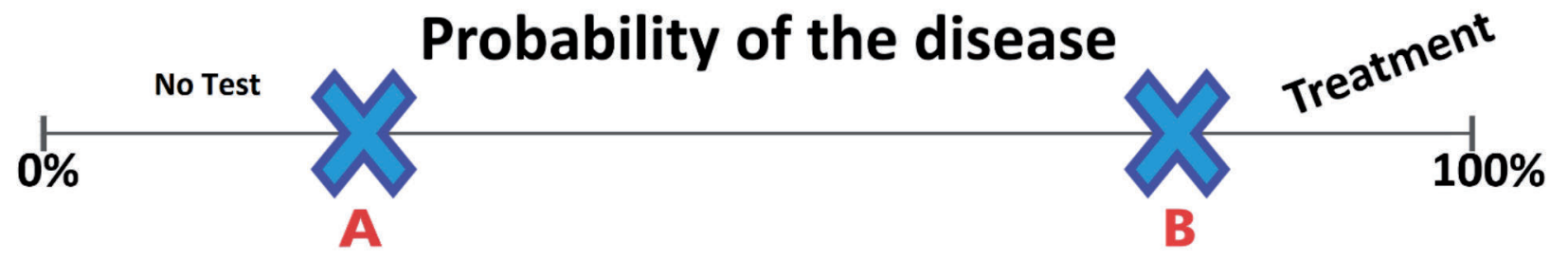

Figure 1. Test and treatment thresholds: A) is the test threshold: below this threshold the probability is so low that no test is suggested; B) treatment threshold: no test is needed we need to start treatment. Tests are only indicated between I and II. 
to rule out a disease, we need a sensitive test. If a sensitive test is positive it rules in disease in a high-risk patient, if a sensitive test is negative it rules out disease in a lowrisk patient.

Alternatively, we can use the likelihood ratio (LR).

\section{Likelihood ratio}

Likelihood ratio express how many times more or less likely a test result is to be found in diseased, compared with non-diseased people. It incorporated both sensitivity and specificity. ${ }^{2}$

probability of a patient with $\mathrm{LR}=\frac{\text { the condition having the test result }}{\text { probability of a patient without }}$.

the condition having the same test result

We have a positive likelihood ratio $(\mathrm{LR}+)$ which is simply made by sensitivity/1-specificity and a negative likelihood ratio (LR-) made by 1 -sensitivity/specificity. The best test to rule in a disease is the one with the largest LR+ while the best test to rule out a disease is the one with the smallest LH-.

We should use a test with the highest $\mathrm{LR}+$ when the test result is positive or the lowest LR- when the test result is negative. The diagnostic strategy involves administering the diagnostic test, determining the post-test probability and decide on further testing or treatment. The test result can either reduce or increase the probability and then we can also repeat the process described above again. Obviously, when choosing a test, other factors, such as cost and availability, must also be considered.
What about when the setting is characterized by low prevalence of disease as in primary care?

For many disease clinicians (and researchers) do not have a single well-validated instrument that can be administered and interpreted easily in a clinical setting. Dementia is a good example. ${ }^{3}$

The administration of a full neuropsychological battery and its interpretation by a neuropsychologist

remains the 'gold standard' for assessing the pattern and the degree of cognitive impairment. ${ }^{4}$ The principal advantage of longer batteries is that these may identify patterns of strengths and weaknesses across multiple functional domains. Unfortunately, the administration of a comprehensive battery is time consuming, expensive, and generally unavailable in most practice settings and absolutely unrealistic in primary care., ${ }^{4,5}$

Diagnostic 'tests' can be considered to include symptoms elicited from the patient's history and signs obtained by physical examination, in addition to laboratory tests and imaging. The low prevalence of many diseases in primary care means that even tests with an exceptionally high sensitivity and specificity have relatively high negative predictive values (NPVs) and low positive predictive values (PPVs). ${ }^{6}$ The high NPV of most tests helps clinicians working in low-prevalence settings, such as General Practitioners (GPs), to correctly identify patients without disease. Conversely, the low PPV of most tests, rather than any lack of knowledge or skill, means that GPs are less able to unequivocally diagnose disease. ${ }^{4,6}$

In clinical decision-making, when disease prevalence is low, although it could appear that we learn more from a negative test result than from a positive test, this is not the case.

\section{What is the point of having a negative test result when we already know that that disease is highly unlikely?}

The main aim for performing a diagnostic test is to gain new information. ${ }^{7-10}$ Test results can have little impact on clinical decision-making when the prevalence of disease is at extremes because there is little difference between the pre- and post-test probabilities $^{7}$ (Figure 1).

\section{Watchful waiting strategy: using time in primary care}

One of the most important diagnostic tasks performed by the primary care physician is to discriminate between the majority of patients with mild, usually self-limited diseases, and the minority with severe diseases. ${ }^{11}$

The test of time involves careful initial assessment of the presenting problem(s), with simple and not time consuming tool, followed by one or more reassessment(s), ideally after a predefined period of time ${ }^{12}$ (Figure 2).

In the reassessment(s), the symptoms or signs may become more clearly defined and may resolve or worsen, or new ones may appear. Using test of time requires a good understanding of the natural course of the common conditions to enable appropriate

Table 2. The role of testing.

1. Rule in a diagnosis

The goal is to confirm that the patient has the disease. We should do this when there is a high pre-test probability.

2. Rule out a diagnosis

The goal is to confirm that the patient does not have the disease. We should do this when there is a low pre-test probability.

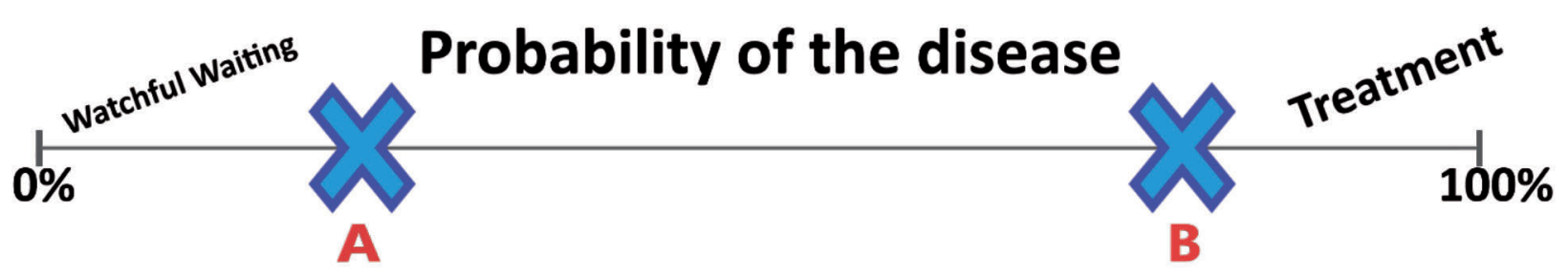

Figure 2. The watchful waiting strategy in primary care: A) is around $10 \%$, below this threshold we should endorse a watchful waiting strategy; B) treatment threshold: no test is needed we need to start treatment. 
reassessments. ${ }^{12,13}$ This enables the clinician to view the disease as an evolving process. ${ }^{14}$

For any test, the relationship between the prevalence and the predictive values is dynamic. ${ }^{15}$ All tests are of their greatest diagnostic use in the 50:50 dilemma when the pre-test probability of the target disease is equally likely to be present or absent. ${ }^{11}$

The time-efficiency principle, on the contrary, operates most effectively at low prevalence, typically between $0 \%$ and $10 \%$ which, after all, is the prevalence of many serious conditions in primary care. (Figure 2). ${ }^{11}$ Here, a small increase in prevalence results in a big decrease in number needed to predict (NNP).

Because most patients without the condition improve within a relatively short time, this creates a dynamic process that increases the prevalence of serious disease in those patients who do not recover in the allotted time. So, the watchful waiting strategy serves as a silent adjudicator-discriminating between those with self-limiting illness and those with serious disease. ${ }^{11,16}$

In Figure 2, the prevalence of serious diseases would increase progressively over time to reach the area of testing (between A and $\mathrm{B}$ ), where any subsequent tests will have their greatest impact on clinical decision-making. The watchful waiting strategy can also have and important effect on how clinicians handle uncertainty. ${ }^{11,17}$

\section{When can the time-efficiency principle be used?}

It is important for clinicians to distinguish between patients who require rapid investigation, treatment, or referral and those who would benefit from serial testing over time. This strategy should only be used in those situations where the benefit of applying time outweighs the risk of harm from a delayed or missed diagnosis, for example if the situation is not immediately life-threatening. ${ }^{11,18}$ By contrast, the occasional patients with red flag symptoms and signs, or who present late, need immediate referral or treatment. ${ }^{12}$

\section{The differences between primary and secondary care}

The main diagnostic challenge in primary care is the low prevalence, and thus the opportunities to use time, are abundant. ${ }^{19}$ Patients are typically seen early in the course of an illness and care should be considered continuous. By contrast, in sec- ondary care, many patients have been through the filter of primary care, where time may have already been used. As a consequence, patients are often seen later in the course of their illness when more urgent, definitive treatment is required.?

\section{Benefits of the watchful waiting strategy}

A major benefit of the test of time is that it serves to protect patients with non-serious disease from unnecessary additional testing. ${ }^{20}$ It is a brake upon cascade effects that occur when an inappropriate test, is followed by a series of tests with increasing momentum, so that stopping becomes progressively harder, possibly moving to sophisticated and expensive tests and procedures where risk exceeds benefits. ${ }^{21}$ Cascade effects can be triggered by inappropriate data gathering such as the erroneous interpretation of a laboratory result outside normal ranges, underestimating the risks of test of treatment, underestimating the possibility of a false-positive result and the intolerance of uncertainty by the doctor. ${ }^{11,17}$ Data from literature show that strong primary care is associated with lower health service costs. ${ }^{14,22,23}$

\section{Requirements to meet for proper use of the test of time}

The effectiveness of the time-efficiency principle is associated with the continuity of personal care provided by the clinician. ${ }^{24}$ For patients to tolerate the inevitable uncertainty of waiting for symptoms to resolve or develop, it is crucial that they are able to trust their GP. ${ }^{24-26}$

A failure to provide such continuity of care weakens the opportunity to use time as a diagnostic strategy.

\section{Threats}

Primary care operates in a relatively technologically unsophisticated environment. The watchful waiting strategy is based on the trust that comes from strong clinician-patient relationships to allow time to be actively used for self-limiting conditions to resolve or pathology to emerge. Over the years, this strategy has been undermined by a growing interest in near-patient diagnostic testing ${ }^{27}$ and the major threats come from patient pressure or defensive practice, as an attempt to minimize uncertainty or avoid litigation..$^{28}$

\section{Conclusions}

Problems are presented to primary care physicians in the community in a very different way from presentations in secondary care, and clinicians often need to reassure anxious patients that the disease is not present.

The challenge for primary care and other low-prevalence settings is to only make diagnoses that are actually useful to patients, at the optimal time, living with the uncertainty of informed doubt. Patients' trust in their doctor should be accompanied by a willingness to tolerate such uncertainty and not rush to technologically interesting investigations before time can be used appropriately to clearly identify the possibility of serious illness. We should avoid that a false reliance on inappropriate, patient-friendly diagnostic tests and the fear of a rare late diagnoses would push us to over-refine diagnoses unnecessarily in advance.

\section{References}

1. Shaneyfelt T. Pyramids are guides not rules: the evolution of the evidence pyramid. Evid Based Med 2016;21:121-2.

2. Parikh R, Parikh S, Arun E, Thomas R. Likelihood ratios: clinical application in day-to-day practice. Indian J Ophthalmol 2009;57:217.

3. Moritz S, Ferahli S, Naber D. Memory and attention performance in psychiatric patients: lack of correspondence between clinician-rated and patient-rated functioning with neuropsychological test results. J Int Neuropsychol Soc 2004;10: 623-33.

4. Petrazzuoli F, Vestberg S, Midlöv P, et al. Brief cognitive tests used in primary care cannot accurately differentiate mild cognitive impairment from subjective cognitive decline. J Alzheim Dis 2020:1-11.

5. Hurford IM, Marder SR, Keefe RS, et al. A brief cognitive assessment tool for schizophrenia: construction of a tool for clinicians. Schizophr Bull 2011;37:538-45.

6. Mathers N, Hodgkin P. The gatekeeper and the wizard: a fairy tale. BMJ 1989; 298:172.

7. Sackett DL, Haynes RB, Tugwell P. Clinical epidemiology: a basic science for clinical medicine. Little: Brown and Company; 1985.

8. Linn S, Grunau PD. New patient-orient- 
ed summary measure of net total gain in certainty for dichotomous diagnostic tests. Epidemiol Perspect Innovat 2006; 3:11.

9. Koepsell TD, Connell FA. Measures of gain in certainty from a diagnostic test. Am J Epidemiol 1985;121:744-53.

10. Salmi LR, McGill U. Re: "Measures of gain in certainty from a diagnostic test". Am J Epidemiol 1986;123:1121-2.

11. Irving G, Holden J. The time-efficiency principle: time as the key diagnostic strategy in primary care. Family Pract 2013;30:386-9.

12. Almond SC, Summerton N. Diagnosis in general practice. Test of time. BMJ 2009;338:b1878.

13. Crossley T. I Don't know what it is but I don't think it's serious: confidence and decisiveness in primary care. London: Royal College of General Practitioners; 2008.

14. Hart JT. The political economy of health care: a clinical perspective; 2006. Available from: https://www.ncbi.nlm. nih.gov/pmc/articles/PMC2465620/

15. Sox Jr HC, Hickman DH, Marton KI, et al. Using the patient's history to estimate the probability of coronary artery disease: a comparison of primary care and referral practices. Am J Med 1990;89:7-14.

16. Soler J-K, Okkes I, Wood M, Lamberts $\mathrm{H}$. The coming of age of ICPC: celebrating the 21st birthday of the International Classification of Primary Care. Family Pract 2008;25:312-7.

17. Schneider A, Löwe B, Barie S, et al. How do primary care doctors deal with uncertainty in making diagnostic decisions? The development of the 'Dealing with Uncertainty Questionnaire'(DUQ). J Evaluat Clin Pract 2010;16:431-7.

18. Hamilton W, Sharp D. Diagnosis of colorectal cancer in primary care: the evidence base for guidelines. Family Pract 2004;21:99-106.

19. Howie J. Diagnosis - the Achilles heel? J R Coll General Pract 1972;22:310.

20. Jamoulle M. Quaternary prevention: first, do not harm. Rev Brasil Med Fam Comun 2015;10:1-3.

21. Mold JW, Stein HF. The cascade effect in the clinical care of patients. Mass Medical Soc 1986;314:512-4.
22. Starfield B. William Pickles Lecture. Primary and specialty care interfaces: the imperative of disease continuity. $\mathrm{Br} \mathrm{J}$ General Pract 2003;53:723.

23. Stange KC, Ferrer RL. The paradox of primary care. Ann Family Med 2009;7: 293-9.

24. Irving G, Reeve J. Do GPs really provide 47 minutes a year for the patient? $\mathrm{Br} \mathrm{J}$ General Pract 2012;62:404-5.

25. Hummers-Pradier E, Beyer M, Chevallier $\mathrm{P}$, et al. The research agenda for general practice/family medicine and primary health care in Europe. Part 1. Background and methodology 1. Eur J General Pract 2009;15:243-50.

26. Hill AP, Freeman GK. Promoting continuity of care in general practice. London: Royal College of General Practitioners; 2011.

27. Rogers E. Diffusion of innovations, 5th edn. New York, NY: Free Press; 2003.

28. Studdert DM, Mello MM, Sage WM, et al. Defensive medicine among high-risk specialist physicians in a volatile malpractice environment. JAMA 2005;293: 2609-17. 